Significant variation in the assessments of control of patient's condition, improvement in sleepiness and compliance after treatment remains (Figure 1). $236 \%$ were not aware that the BTS have issued a statement $63 \%$ felt the change in emphasis from excessive sleepiness to sleepiness likely to impair safe driving helpful. 64\% of respondents were not aware that DVLA had changed its guidance in January 2016. 18\% of respondents advise patients to inform the DVLA when diagnosis felt to be likely based on symptoms. 57\% when diagnosis confirmed following investigation, $13 \%$ when CPAP first trialled and 12\% when CPAP issued to the patient.

Conclusions The results of the 2016 survey confirm the results of the 2013 survey. Disappointingly the guidance from the BTS appears to have had little impact. The change in emphasis from excessively sleepy to sleepiness likely to impair safe driving was felt to be helpful by a small majority. There is a clear need for tools which are felt to be robust by clinicians and patients to help make decisions about fitness to drive and for these to be disseminated to clinicians.

\section{P66 FALLING ASLEEP WHILE DRIVING: IS DRIVING SAFETY ADVICE GIVEN TO PATIENTS WITH EXCESSIVE DAYTIME SLEEPINESS?}

A Khetarpal, K Anderson, S West. Regional Sleep Service, Newcastle upon Tyne Hospitals, Newcastle upon Tyne, UK

\subsection{6/thoraxjnl-2016-209333.209}

Background 3.5 million people in the UK have excessive daytime sleepiness and 1 in 5 Road Traffic Accidents are due to sleepiness while driving.

Aim To improve Patient and Public safety by auditing whether DVLA's driving safety advice is given to patients with excessive daytime sleepiness in two settings: (i) at referral as recommended by The Royal Society for the Prevention of Accidents guidelines and (ii) at the Regional Sleep Clinic as recommended by the BTS guidelines.

Method Retrospective study between 01/10/15 and 06/01/16 of (i) 100 referral letters to the Regional Sleep Clinic and (ii) 100 sleep clinic letters to patients' GP. In both cases, patients were included if the letter mentioned 'Daytime sleepiness' or if their Epworth Sleepiness Score (ESS) was over 10 (indicating excessive daytime sleepiness).

Results Only 19\% of referral letters from primary and secondary care had documented giving driving safety advice to patients with daytime sleepiness. Sixteen specialties referred patients to the Sleep clinic. General Practice accounted for three quarters of these referrals and driving safety had only been discussed in 14\% of cases. Even with specialties like Respiratory medicine and Neurology which see patients with sleep disorders regularly, very few had discussed driving safety.

The Sleep Clinic gave DVLA advice to $85 \%$ of patients. In the $15 \%$ where no advice was given, patients usually had ESS $<10$ (but symptomatically sleepy) or sleepiness as a secondary consequence of insomnia/non-REM parasomnia.

7 patients reported falling asleep while driving (only 2/7 were discovered at referral). Moreover, the Sleep Clinic noted that 1 had a Road Traffic Accident and 1 had a near miss. Average waiting time from referral to Sleep Clinic appointment was 3 months. Thus driving advice needs to be given at referral.
A limitation of this audit is that not all clinicians record discussions about driving even though it is important for medicolegal purposes.

Conclusion Driving safety discussions on referral can be improved by educating GPs/secondary care and introducing an Alert on eReferral. The Sleep Clinic should use a pro forma to remind clinicians to discuss driving regardless of a patient's ESS or diagnosis.

\section{P67 IS THERE A DIFFERENCE BETWEEN THE SLEEP PHYSIOLOGY OF OBESE AND SUPER OBESE PATIENTS?}

A Rajhan, L Michael, A Bain, A Thomas, M Allen. University Hospital of North Midlands, Stoke-On-Trent, UK

\subsection{6/thoraxjnl-2016-209333.210}

Introduction Bariatric surgery is increasingly recommended for managing patients who are both obese and super obese (BMI $\geq 45$ ref. WHO Classification). We have compared if there are physiological and subjective differences between patients in these two categories.

Methods Patients assessed for bariatric surgery were split into the super obese and obese group. Their physiological parameters including Apnoea Hypopnoea Index(AHI), Desaturation Index (ODI $>4 \%$ ) and subjective results i.e., Epworth Sleepiness Score (ESS) and STOPBANG Questionnaire were compared.

Results 111 patients assessed for bariatric surgery attended for limited sleep studies from a period between July 2013 to December 2014. 57 patients were obese (40 females) and 54 were superobese (37 females) and the results are tabulated below. (Table 1)

The superobese patients had a higher AHI, ODI and time spent desaturated when compared to the obese patients.

Conclusion 1) There is more physiological derangement in the super obese patient group so greater caution is needed in the administration of anaesthetic to such patients.

2) Despite the physiological derangement, superobese patients were less sleepy based on their ESS, the reasons for which are not entirely clear.

\begin{tabular}{llllll}
\multicolumn{6}{l}{ Abstract P67 Table 1 } \\
\hline \multicolumn{5}{l}{ Obese $(\mathbf{n}=\mathbf{5 7})$} & \multicolumn{4}{l}{ Super obese $(\mathbf{n}=\mathbf{5 4})$} & p value \\
\hline & Mean & SD & Mean & SD & \\
ODI $>$ 4\% & 20.64 & 18.78 & 31.46 & 26.53 & 0.024 \\
Time spent $<90 \%$ & 8.03 & 14.78 & 12.93 & 17.65 & 0.012 \\
Time spent $<85 \%$ & 1.85 & 7.66 & 2.99 & 5.615 & 0.007 \\
AHI & 21.71 & 19.67 & 31.49 & 27.11 & 0.047 \\
ESS & 8.85 & 5.2 & 6.72 & 4.77 & 0.035 \\
STOPBANG & Median - 5 & IQR 4-6 & Median - 5 & IQR 4-6 & 0.22 \\
Mallampatti Score & Median - 3 & IQR 2-4 & Median - 3 & IQR 1-4 & 0.711 \\
\hline SD - Standard Deviation IQR - Interquartile Range & & &
\end{tabular}

\section{\begin{tabular}{|l|l} 
P68 TO SCREEN OR NOT TO SCREEN FOR OBSTRUCTIVE \\
\hline
\end{tabular} SLEEP APNOEA (OSA) PRE-OPERATIVELY?}

${ }^{1} \mathrm{CD}$ Turnbull, ${ }^{2} \mathrm{D}$ Ball, ${ }^{3} \mathrm{ML}$ Estevez, ${ }^{2} \mathrm{H}$ Du Plessis, ${ }^{1} \mathrm{M}$ Hardinge, ${ }^{1} \mathrm{~A}$ Nickol. ${ }^{1} \mathrm{O}$ xford Centre for Respiratory Medicine, Oxford University Hospitals NHS Foundation Trust, UK; ${ }^{2}$ Department of Anaesthetics, Oxford University Hospitals NHS Foundation Trust, UK; ${ }^{3}$ Hospital Clinico San Carlos, Madrid, Spain 